\title{
Symmetry energy of deformed neutron-rich nuclei
}

\author{
M. K. Gaidarov, ${ }^{1}$ A. N. Antonov, ${ }^{1}$ P. Sarriguren, ${ }^{2}$ and E. Moya de Guerra ${ }^{3}$ \\ ${ }^{1}$ Institute for Nuclear Research and Nuclear Energy, Bulgarian Academy of Sciences, Sofia 1784, Bulgaria \\ ${ }^{2}$ Instituto de Estructura de la Materia, IEM-CSIC, Serrano 123, E-28006 Madrid, Spain \\ ${ }^{3}$ Departamento de Fisica Atomica, Molecular y Nuclear, Facultad de Ciencias Fisicas, Universidad Complutense de Madrid, \\ E-28040 Madrid, Spain
}

(Received 17 April 2012; published 21 June 2012)

\begin{abstract}
The symmetry energy, the neutron pressure, and the asymmetric compressibility of deformed neutron-rich even-even nuclei are calculated for the examples of $\mathrm{Kr}$ and $\mathrm{Sm}$ isotopes within the coherent density fluctuation model using the symmetry energy as a function of density within the Brueckner energy-density functional. The correlation between the thickness of the neutron skin and the characteristics related to the density dependence of the nuclear symmetry energy is investigated for isotopic chains of these nuclei in the framework of the self-consistent Skyrme-Hartree-Fock plus BCS method. Results for an extended chain of $\mathrm{Pb}$ isotopes are also presented. A remarkable difference is found in the trend followed by the different isotopic chains: the studied correlations reveal a smoother behavior in the $\mathrm{Pb}$ case than in the other cases. We also notice that the neutron skin thickness obtained for ${ }^{208} \mathrm{~Pb}$ with the SLy4 force is found to be in a good agreement with recent data.
\end{abstract}

DOI: 10.1103/PhysRevC.85.064319

PACS number(s): 21.60.Jz, 21.65.Ef, 21.10.Gv

\section{INTRODUCTION}

The study of the nuclear matter symmetry energy that essentially characterizes the isospin-dependent part of the equation of state (EOS) of asymmetric nuclear matter (ANM) is currently an exciting topic of research in nuclear physics [1-4]. In fact, applications of ANM are broad, ranging from the structure of rare isotopes $[5,6]$ to the properties of neutron stars $[7,8]$ and the dynamical process of nuclear reactions [9]. The transition from ANM to finite nuclei is a natural and important way to learn more about the nuclear symmetry energy, which is poorly constrained by experimental data on ground-state nuclear properties.

The ground states of atomic nuclei are characterized by different equilibrium configurations related to corresponding geometrical shapes. The study of the latter, as well as the transition regions between them, has been a subject of a large number of theoretical and experimental studies (for a review, see, for example, Ref. [10] and references therein). The position of the neutron drip line is closely related to the neutron excess and the deformation in nuclei. Deformed nuclei are expected in several regions near the neutron drip line [11,12]. In some cases, the deformation energy can impact their existence. For instance, it has been predicted that there exist particle-bound even-even nuclei that have, at the same time, negative two-neutron separation energies caused by shape coexistence effects [11]. In fact, the nuclear deformation increases the surface area, thus leading to a larger surface symmetry energy in a neutron-rich nucleus with a deformed shape. Conversely, the precise determination of the surface symmetry energy is important to describe the deformability of neutron-rich systems and also to validate theoretical extrapolations.

The theoretical treatment of the ground-state properties of deformed nuclei is usually made in the framework of mean-field approaches; for instance, the nonrelativistic self-consistent Hartree-Fock + BCS approach with density-dependent Skyrme interaction [13-17], or the HartreeFock-Bogoliubov (HFB) approximation that uses, in particular, the Gogny force containing a finite-range interaction $[18,19]$. Also the relativistic mean-field (RMF) models with different types of parameter sets have been widely used. For instance, the RMF theory with parameter set FSUGold has been recently employed to study the binding energies, quadrupole deformations, charge radii, and neutron skins of rare-earth even-even nuclei ranged from $Z=58$ to $Z=70$ and some deformed nuclei (Nd, Sm, Gd, and Dy) in other regions [20]. This new parameter set that includes the nonlinear coupling between the isoscalar and isovector mesons is shown to reproduce successfully the ground-state properties of deformed nuclei. Also, the same parameter set FSUGold can successfully reflect the shell effect of the neutron magic number $N=82$, thus leading to a good description of the ground-state properties of $\mathrm{Sn}, \mathrm{Te}, \mathrm{Xe}$, and $\mathrm{Ba}$ isotopes [21].

Nowadays, the experimental information about the symmetry energy is fairly limited. The need to have information for this quantity in finite nuclei, even theoretically obtained, is a major issue because it allows one to constrain the bulk and surface properties of the nuclear energy-density functionals (EDFs) quite effectively. For example, the traditional Skyrme EDF based on the leptodermous expansion of the smooth nuclear energy was used in Ref. [22], where the strongly correlated symmetry and surface symmetry terms in this expansion are resolved by considering data on deformed neutron-rich nuclei in which the surface symmetry term is amplified. By starting from microscopic nucleon self-energies in nuclear matter, the authors of Ref. [23] have made a comprehensive study of deformation properties of relativistic nuclear EDFs for a set of 64 axially deformed nuclei in the mass regions $A \approx 150-180$ and $A \approx 230-250$.

In our recent work [24] the Brueckner EDF for infinite nuclear matter was applied to calculate nuclear quantities of medium-heavy and heavy $\mathrm{Ni}, \mathrm{Sn}$, and $\mathrm{Pb}$ nuclei that include surface effects, namely the nuclear symmetry energy 
$s$, the neutron pressure $p_{0}$, and the asymmetric compressibility $\Delta K$. For this purpose, a theoretical approach that combines the deformed HF + BCS method with Skyrme-type densitydependent effective interactions [13] and the coherent density fluctuation model (CDFM) $[25,26]$ was used. We would like to note the capability of the CDFM to be applied as an alternative way to make a transition from the properties of nuclear matter to the properties of finite nuclei. We have found that there exists an approximate linear correlation between the neutron skin thickness $\Delta R$ of even-even nuclei from the $\mathrm{Ni}(A=74-84)$, $\mathrm{Sn}(A=124-152)$, and $\mathrm{Pb}(A=206-214)$ isotopic chains and their nuclear symmetry energies. A similar linear correlation between $\Delta R$ and $p_{0}$ was also found to exist, while the relation between $\Delta R$ and $\Delta K$ turned out to be less pronounced. The kinks displayed by Ni and Sn isotopes and the lack of such a kink in the $\mathrm{Pb}$ chain considered [24] were shown to be mainly due to the shell structure of these exotic nuclei, but they deserve further analysis within the used theoretical approach.

Another interesting question is to explore how the nuclear symmetry energy changes in the presence of deformation and correlates with the neutron skin thickness within a given isotopic chain. In Ref. [16], the effects of deformation on the skin formation were studied in $\mathrm{Kr}$ isotopes that are well deformed nuclei. It has been shown from the analysis on ${ }^{98,100} \mathrm{Kr}$ nuclei that although the profiles of the proton and neutron densities, as well as the spatial extensions, change with the direction in both oblate and prolate shapes, the neutron skin thickness remains almost equal along the different directions perpendicular to the surface. Thus, a very weak dependence of the neutron skin formation on the character of the deformation was found [16].

In the present work an investigation of possible relation between the neutron skin thickness and the basic nuclear matter properties in deformed finite nuclei, such as the symmetry energy at the saturation point, symmetry pressure, and asymmetric compressibility, is carried out for chains of deformed neutron-rich even-even $\operatorname{Kr}(A=82-96)$ (including, as well, the case of some extreme neutron-rich nuclei up to ${ }^{120} \mathrm{Kr}$ ) and $\mathrm{Sm}(A=140-156)$ isotopes, following the theoretical method of Ref. [24]. We also present for comparison results for an extended chain of $\mathrm{Pb}(A=202-214)$ isotopes. This is motivated by the significant interest (in both experiment [27-29] and theory [30-33]) to study the neutron distribution and rms radius in ${ }^{208} \mathrm{~Pb}$, aiming at precise determinations of the neutron skin in this nucleus. In addition to the interest that this study may have by itself as well as in combination with the previous calculations of Ref. [24], we give some numerical arguments in proof of the existence of kinks in $\mathrm{Ni}$ and $\mathrm{Sn}$ isotopic chains that are not present in the $\mathrm{Pb}$ chain. The kinks are produced because of the sensitivity of the symmetry energy and neutron pressure to the shell structure (see, for instance, the discussion in Refs. [24,34,35]).

The paper is organized as follows. In Sec. II we present a brief description of the theoretical formalism (definitions of ANM properties, CDFM basic expressions, Brueckner energy-density functional, and Hartree-Fock + BCS densities) used to unveil a possible correlation between the neutron skin thickness and the nuclear matter characteristics of the considered isotopic chains. Section III contains our results with a discussion on the obtained relationships and on the presence of kinks. Concluding remarks are given in Sec. IV.

\section{THEORETICAL FRAMEWORK}

We study in the present work the symmetry energy $s(\rho)$ and related quantities of finite deformed nuclei on the basis of the corresponding definitions for ANM. The quantity $s^{\mathrm{ANM}}(\rho)$, which refers to the infinite system and therefore neglects surface effects, is related to the second derivative of the energy per particle $E(\rho, \delta)$ using its Taylor series expansion in terms of the isospin asymmetry $\delta=\left(\rho_{n}-\rho_{p}\right) / \rho\left(\rho, \rho_{n}\right.$, and $\rho_{p}$ being the baryon, neutron and proton densities, respectively) (see, e.g., Refs. [24,36,37]):

$$
\begin{aligned}
s^{\mathrm{ANM}}(\rho)= & \left.\frac{1}{2} \frac{\partial^{2} E(\rho, \delta)}{\partial \delta^{2}}\right|_{\delta=0}=a_{4}+\frac{p_{0}^{\mathrm{ANM}}}{\rho_{0}^{2}}\left(\rho-\rho_{0}\right) \\
& +\frac{\Delta K^{\mathrm{ANM}}}{18 \rho_{0}^{2}}\left(\rho-\rho_{0}\right)^{2}+\cdots
\end{aligned}
$$

In Eq. (1) the parameter $a_{4}$ is the symmetry energy at equilibrium $\left(\rho=\rho_{0}\right)$. In ANM the pressure $p_{0}^{\text {ANM }}$ and the curvature $\Delta K^{\mathrm{ANM}}$ are

$$
\begin{gathered}
p_{0}^{\mathrm{ANM}}=\left.\rho_{0}^{2} \frac{\partial s^{\mathrm{ANM}}(\rho)}{\partial \rho}\right|_{\rho=\rho_{0}}, \\
\Delta K^{\mathrm{ANM}}=\left.9 \rho_{0}^{2} \frac{\partial^{2} s^{\mathrm{ANM}}(\rho)}{\partial \rho^{2}}\right|_{\rho=\rho_{0}} .
\end{gathered}
$$

The "slope" parameter $L^{\mathrm{ANM}}$ is defined as

$$
L^{\mathrm{ANM}}=\frac{3 p_{0}^{\mathrm{ANM}}}{\rho_{0}} .
$$

In general, the predictions for the symmetry energy vary quite substantially: e.g., $a_{4} \equiv s\left(\rho_{0}\right)=28-38 \mathrm{MeV}$ while an empirical value of $a_{4} \approx 29 \mathrm{MeV}$ has been extracted from finite nuclei by fitting the ground-state energies using the generalized Weizsäcker mass formula (see, e.g., Ref. [38]). By using the experimental pygmy strength, an average value of $a_{4}=32.0 \pm 1.8 \mathrm{MeV}$ was obtained from the ${ }^{130,132} \mathrm{Sn}$ analysis [39], which is within the acceptable range of values of $a_{4}$ to be around $32.5 \mathrm{MeV}$ coming from various experiments using different experimental probes (for a recent status, see, for example, Ref. [40] and references therein).

In Ref. [24] we calculated the symmetry energy, the pressure and slope, as well as the curvature for finite nuclei applying the coherent density fluctuation model (suggested and developed in Refs. $[25,26])$. In the CDFM the one-body density matrix $\rho\left(\mathbf{r}, \mathbf{r}^{\prime}\right)$ of the nucleus is written as a coherent superposition of the one-body density matrices $\rho_{x}\left(\mathbf{r}, \mathbf{r}^{\prime}\right)$ for spherical "pieces" of nuclear matter called "fluctons" with densities $\rho_{x}(\mathbf{r})=\rho_{0}(x) \Theta(x-|\mathbf{r}|), \rho_{0}(x)=3 A / 4 \pi x^{3}$ :

$$
\rho\left(\mathbf{r}, \mathbf{r}^{\prime}\right)=\int_{0}^{\infty} d x|f(x)|^{2} \rho_{x}\left(\mathbf{r}, \mathbf{r}^{\prime}\right)
$$


with

$$
\begin{aligned}
\rho_{x}\left(\mathbf{r}, \mathbf{r}^{\prime}\right)= & 3 \rho_{0}(x) \frac{j_{1}\left(k_{F}(x)\left|\mathbf{r}-\mathbf{r}^{\prime}\right|\right)}{\left(k_{F}(x)\left|\mathbf{r}-\mathbf{r}^{\prime}\right|\right)} \\
& \times \Theta\left(x-\frac{\left|\mathbf{r}+\mathbf{r}^{\prime}\right|}{2}\right),
\end{aligned}
$$

where $j_{1}$ is the first-order spherical Bessel function,

$$
k_{F}(x)=\left(\frac{3 \pi^{2}}{2} \rho_{0}(x)\right)^{1 / 3} \equiv \frac{\alpha}{x}
$$

with

$$
\alpha=\left(\frac{9 \pi A}{8}\right)^{1 / 3} \simeq 1.52 A^{1 / 3}
$$

is the Fermi momentum of the nucleons in the flucton with a radius $x$. In Eq. (5) $|f(x)|^{2}$ is the weight function that in the case of monotonically decreasing local densities $[d \rho(r) / d r \leqslant$ $0]$ can be obtained using a known density distribution for a given nucleus:

$$
|f(x)|^{2}=-\left.\frac{1}{\rho_{0}(x)} \frac{d \rho(r)}{d r}\right|_{r=x}
$$

with the normalization $\int_{0}^{\infty} d x|f(x)|^{2}=1$.

The main assumption of the CDFM is that properties of finite nuclei can be calculated using the corresponding ones for nuclear matter, folding them with the weight function $|f(x)|^{2}$. Along this line, in the CDFM the symmetry energy for finite nuclei and related quantities are assumed to be infinite superpositions of the corresponding ANM quantities weighted by $|f(x)|^{2}$ :

$$
\begin{aligned}
s & =\int_{0}^{\infty} d x|f(x)|^{2} s^{\mathrm{ANM}}(x), \\
p_{0} & =\int_{0}^{\infty} d x|f(x)|^{2} p_{0}^{\mathrm{ANM}}(x), \\
\Delta K & =\int_{0}^{\infty} d x|f(x)|^{2} \Delta K^{\mathrm{ANM}}(x) .
\end{aligned}
$$

The explicit forms of the ANM quantities $s^{\mathrm{ANM}}(x), p_{0}^{\mathrm{ANM}}(x)$, and $\Delta K^{\mathrm{ANM}}(x)$ in Eqs. (10)-(12) are defined below. They have to be determined within a chosen method for description of the ANM characteristics. In the present work, as well as in Ref. [24], considering the pieces of nuclear matter with density $\rho_{0}(x)$, we use for the matrix element $V(x)$ of the nuclear Hamiltonian the corresponding ANM energy from the method of Brueckner et al. [41,42]:

$$
V(x)=A V_{0}(x)+V_{C}-V_{C O},
$$

where

$$
\begin{aligned}
V_{0}(x)= & 37.53\left[(1+\delta)^{5 / 3}+(1-\delta)^{5 / 3}\right] \rho_{0}^{2 / 3}(x) \\
& +b_{1} \rho_{0}(x)+b_{2} \rho_{0}^{4 / 3}(x)+b_{3} \rho_{0}^{5 / 3}(x) \\
& +\delta^{2}\left[b_{4} \rho_{0}(x)+b_{5} \rho_{0}^{4 / 3}(x)+b_{6} \rho_{0}^{5 / 3}(x)\right]
\end{aligned}
$$

with

$$
\begin{array}{ll}
b_{1}=-741.28, & b_{2}=1179.89, \\
b_{4}=148.26, & b_{3}=-467.54, \\
b_{5}=372.84, & b_{6}=-769.57 .
\end{array}
$$

In Eq. (13) $V_{0}(x)$ is the energy per particle in nuclear matter (in $\mathrm{MeV}$ ) accounting for the neutron-proton asymmetry, $V_{C}$ is the Coulomb energy of protons in a flucton,

$$
V_{C}=\frac{3}{5} \frac{Z^{2} e^{2}}{x},
$$

and $V_{C O}$ is the Coulomb exchange energy:

$$
V_{C O}=0.7386 Z e^{2}\left(3 Z / 4 \pi x^{3}\right)^{1 / 3}
$$

Thus, using the Brueckner theory, the symmetry energy $s^{\mathrm{ANM}}(x)$ and the related quantities for ANM with density $\rho_{0}(x)$ [the coefficient $a_{4}$ in Eq. (1)] have the forms

$$
\begin{aligned}
s^{\mathrm{ANM}}(x)= & 41.7 \rho_{0}^{2 / 3}(x)+b_{4} \rho_{0}(x) \\
& +b_{5} \rho_{0}^{4 / 3}(x)+b_{6} \rho_{0}^{5 / 3}(x), \\
p_{0}^{\mathrm{ANM}}(x)= & 27.8 \rho_{0}^{5 / 3}(x)+b_{4} \rho_{0}^{2}(x) \\
& +\frac{4}{3} b_{5} \rho_{0}^{7 / 3}(x)+\frac{5}{3} b_{6} \rho_{0}^{8 / 3}(x),
\end{aligned}
$$

and

$$
\Delta K^{\mathrm{ANM}}(x)=-83.4 \rho_{0}^{2 / 3}(x)+4 b_{5} \rho_{0}^{4 / 3}(x)+10 b_{6} \rho_{0}^{5 / 3}(x) .
$$

In our method (see also Ref. [24]) Eqs. (18)-(20) are used to calculate the corresponding quantities in finite nuclei $s, p_{0}$, and $\Delta K$ from Eqs. (10)-(12), respectively. We note that in the limit case when $\rho(r)=\rho_{0} \Theta(R-r)$ and $|f(x)|^{2}$ becomes a $\delta$ function [see Eq. (9)], Eq. (10) reduces to $s^{\mathrm{ANM}}\left(\rho_{0}\right)=a_{4}$.

In our work we use the proton and neutron densities obtained from self-consistent deformed Hartree-Fock calculations with density-dependent Skyrme interactions [13] and pairing correlations. Pairing between like nucleons is included by solving the BCS equations at each iteration with a fixed pairing strength that reproduces the odd-even experimental mass differences [43].

The spin-independent proton and neutron densities are given by $[16,44]$

$$
\rho(\vec{R})=\rho(r, z)=\sum_{i} 2 v_{i}^{2} \rho_{i}(r, z),
$$

where $r$ and $z$ are the cylindrical coordinates of $\vec{R}, v_{i}^{2}$ are the occupation probabilities resulting from the BCS equations, and $\rho_{i}$ are the single-particle densities

$$
\rho_{i}(\vec{R})=\rho_{i}(r, z)=\left|\Phi_{i}^{+}(r, z)\right|^{2}+\left|\Phi_{i}^{-}(r, z)\right|^{2}
$$

with

$$
\Phi_{i}^{ \pm}(r, z)=\frac{1}{\sqrt{2 \pi}} \sum_{\alpha} \delta_{\Sigma, \pm 1 / 2} \delta_{\Lambda, \Lambda^{\mp}} C_{\alpha}^{i} \psi_{n_{r}}^{\Lambda}(r) \psi_{n_{z}}(z)
$$


and $\alpha=\left\{n_{r}, n_{z}, \Lambda, \Sigma\right\}$. In Eq. (23) the functions $\psi_{n_{r}}^{\Lambda}(r)$ and $\psi_{n_{z}}(z)$ are expressed by Laguerre and Hermite polynomials:

$$
\begin{aligned}
& \psi_{n_{r}}^{\Lambda}(r)=\sqrt{\frac{n_{r}}{\left(n_{r}+\Lambda\right) !}} \beta_{\perp} \sqrt{2} \eta^{\Lambda / 2} e^{-\eta / 2} L_{n_{r}}^{\Lambda}(\eta), \\
& \psi_{n_{z}}(z)=\sqrt{\frac{1}{\sqrt{\pi} 2^{n_{z}} n_{z} !}} \beta_{z}^{1 / 2} e^{-\xi^{2} / 2} H_{n_{z}}(\xi)
\end{aligned}
$$

with

$$
\begin{aligned}
& \beta_{z}=\left(m \omega_{z} / \hbar\right)^{1 / 2}, \quad \beta_{\perp}=\left(m \omega_{\perp} / \hbar\right)^{1 / 2}, \\
& \xi=z \beta_{z}, \quad \eta=r^{2} \beta_{\perp}^{2} .
\end{aligned}
$$

The normalization of the densities is

$$
\int \rho(\vec{R}) d \vec{R}=X
$$

with $X=Z, N$ for protons and neutrons, respectively.

The multipole decomposition of the density can be written in terms of even $\lambda$ multipole components as $[13,44]$

$$
\rho(r, z)=\sum_{\lambda} \rho_{\lambda}(R) P_{\lambda}(\cos \theta)
$$

In the calculations, for the density distribution $\rho(r)$ needed to obtain the weight function $|f(x)|^{2}$ [Eq. (9)], we use the monopole term $\rho_{0}(R)$ in the expansion (28).

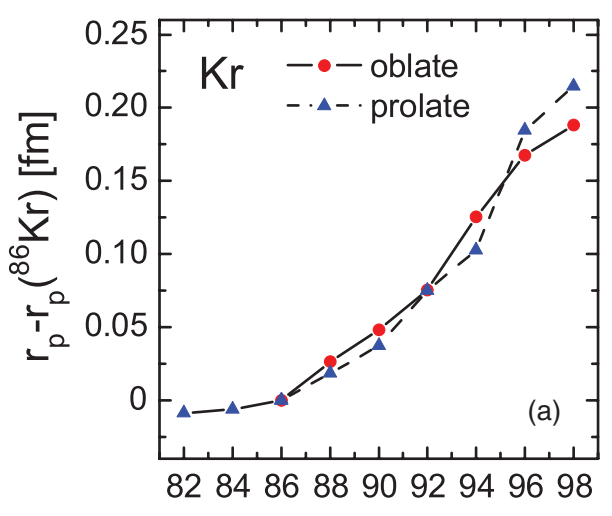

A
The neutron skin thickness is usually estimated as the difference of the rms radii of neutrons and protons:

$$
\Delta R=\left\langle r_{\mathrm{n}}^{2}\right\rangle^{1 / 2}-\left\langle r_{\mathrm{p}}^{2}\right\rangle^{1 / 2}
$$

In our calculations the following Skyrme force parametrizations are used: SLy4 [45], Sk3 [46], SGII [47], and LNS [48]. These are among the most extensively used Skyrme forces that work successfully for describing finite nuclei properties. Although it is well known that SGII and LNS interactions do not predict accurate binding energies in finite nuclei, we have included them in our work because they are representative examples of Skyrme interactions and because we are not concerned here with the absolute values of binding energies, but rather with the isotopic evolution of relative differences of various magnitudes and energy derivatives.

\section{RESULTS AND DISCUSSION}

We start our analysis by searching for the role of deformation on the rms radii. It is illustrated by showing the differences between the proton (neutron) radii and the corresponding proton (neutron) radius of semimagic ${ }^{86} \mathrm{Kr}(N=50)$ which is taken as a reference nucleus. The results are given in Figs. 1(a) and 1(b), respectively. In addition, in Fig. 1(c) our results with the SLy4 force for the squared charge radii differences

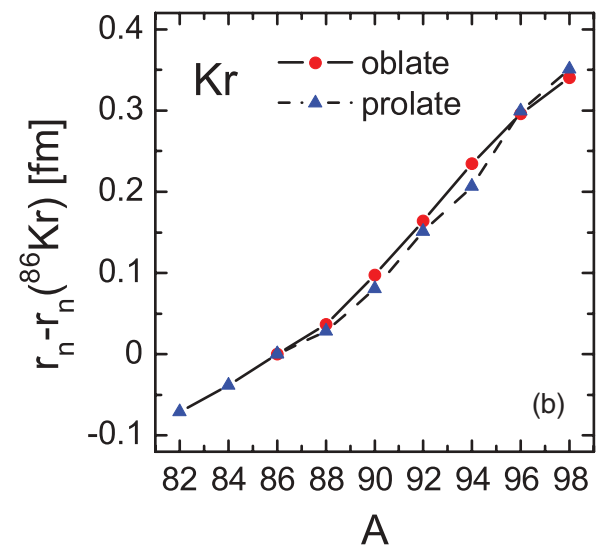

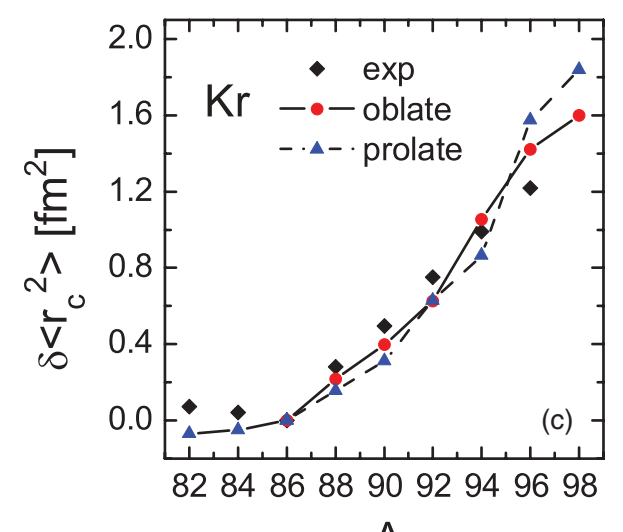

A

FIG. 1. (Color online) Proton (a) and neutron (b) rms radii differences relative to ${ }^{86} \mathrm{Kr}$ for oblate and prolate nuclear shapes. (c) Theoretical (with the SLy4 Skyrme force) and experimental isotope shifts $\delta\left\langle r_{c}^{2}\right\rangle$ of $\mathrm{Kr}$ isotopes relative to ${ }^{86} \mathrm{Kr}$. The results for oblate and prolate shape for $A=82,84$ isotopes are indistinguishable. 

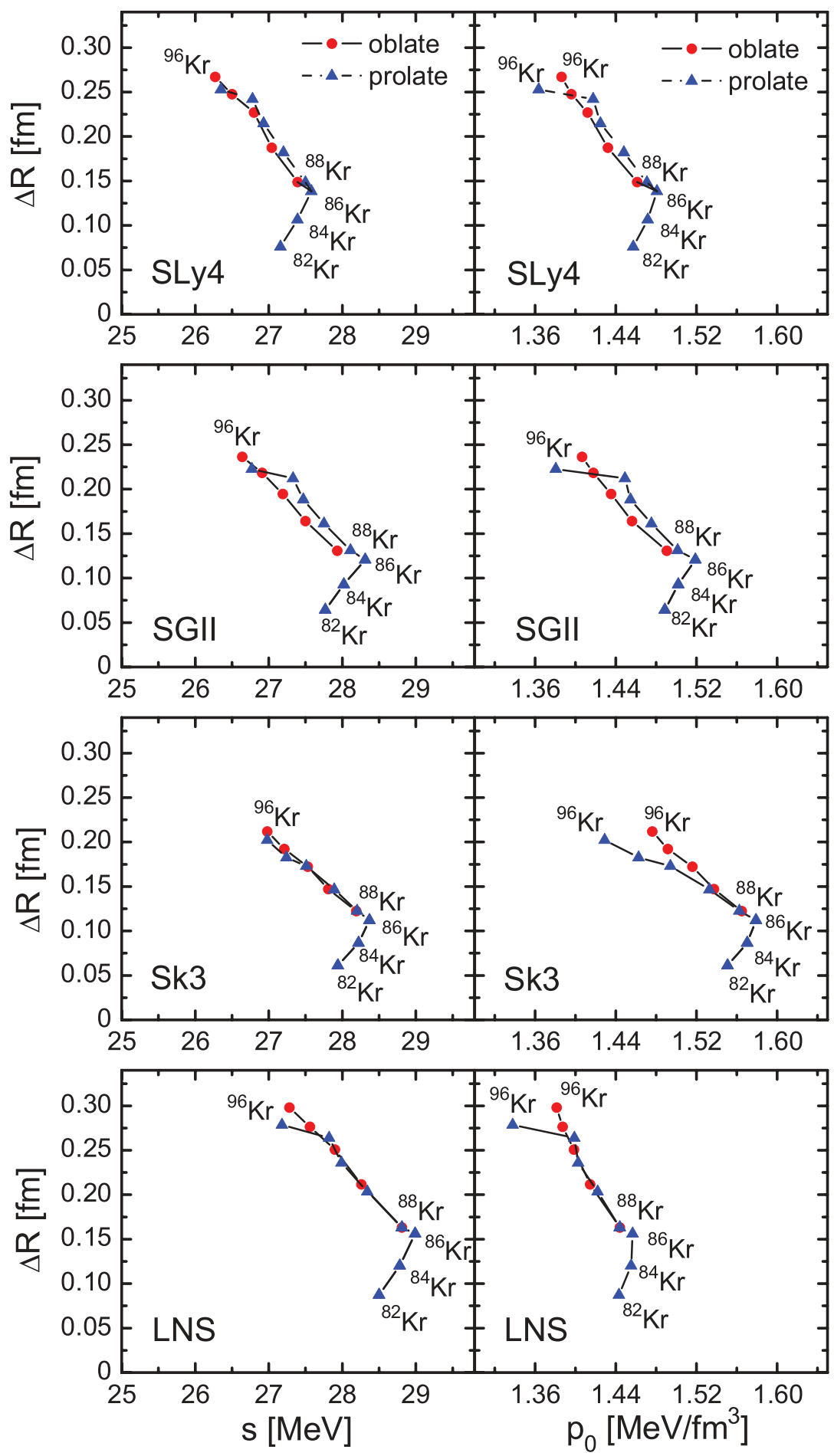

FIG. 2. (Color online) HF + BCS neutron skin thicknesses $\Delta R$ for $\mathrm{Kr}$ isotopes as a function of the symmetry energy $s$ and the pressure $p_{0}$ calculated with SLy4, SGII, Sk3, and LNS forces and for oblate and prolate shapes. The results for oblate and prolate shape for $A=82,84$ isotopes are indistinguishable. in $\mathrm{Kr}$ isotopes are shown, and they are compared with the experimental data from Ref. [49]. Results of this type have been presented in Ref. [16], where the charge radii differences in Sn isotopes obtained from SLy4, SGII, and Sk3 Skyrme forces are compared with the experimental data, taking the radius of ${ }^{120} \mathrm{Sn}$ as the reference. In principle, these differences have been found to be very sensitive probes of nuclear shape transitions, and it is worth studying globally a wide region of neutron-rich exotic nuclei and discussing the similarities and differences among the various isotopic chains [50].
In this analysis the even-even ${ }^{86} \mathrm{Kr}$ isotope turns out to be spherical, while for $\mathrm{Kr}$ isotopes beyond the semimagic ${ }^{86} \mathrm{Kr}$ $(N=50)$ nucleus a deformation (oblate or prolate) takes place. We note that for the considered lightest isotopes with $A=82$ and 84 , both shapes produce results that are indistinguishable and, therefore, only the prolate solutions are presented. A smooth increase of radii differences relative to ${ }^{86} \mathrm{Kr}$ with increasing neutron number can be observed in all three panels of Fig. 1. The charge and proton radii grow following the growth of the neutron radius with increasing neutron number. 
These increases are only slightly dependent on whether an oblate or a prolate shape is considered. A similar behavior has been obtained from Gogny-D1S-HFB calculations performed in Ref. [50]. A satisfactory agreement with the experimental isotope shifts is observed in Fig. 1(c) that provides a good starting point to study further quantities such as the symmetry energy and related characteristics of deformed nuclei within our theoretical method.

Next, an illustration of a possible correlation of the neutron skin thickness $\Delta R$ with the s and $p_{0}$ parameters, extracted from the density dependence of the symmetry energy around the saturation density for the $\mathrm{Kr}$ isotopic chain, is given in Fig. 2. The symmetry energy and the pressure are calculated within the CDFM according to Eqs. (10) and (11) by using the weight functions (9) calculated from the self-consistent densities in Eq. (21). The differences between the neutron and proton rms radii of these isotopes [Eq. (29)] are obtained from HF + BCS calculations using four different Skyrme forces, SLy4, SGII, Sk3, and LNS. It can be seen from Fig. 2 that there exists an approximate linear correlation between $\Delta R$ and $s$ for the eveneven $\mathrm{Kr}$ isotopes with $A=82-96$. Similarly to the behavior of $\Delta R$ vs $s$ dependence for the cases of $\mathrm{Ni}$ and $\mathrm{Sn}$ isotopes [24], we observe a smooth growth of the symmetry energy up to the semimagic nucleus ${ }^{86} \mathrm{Kr}(N=50)$ and then a linear decrease of $s$ while the neutron skin thickness of the isotopes increases. This linear tendency expressed for $\mathrm{Kr}$ isotopes with $A>86$ is similar for the cases of both oblate and prolate deformed shapes. We note that all Skyrme parametrizations used in the calculations reveal similar behavior; in particular, the average slope of $\Delta R$ for various forces is almost the same.

In addition, one can see from Fig. 2 a stronger deviation between the results for oblate and prolate shape of $\mathrm{Kr}$ isotopes in the case of SGII parametrization when displaying the correlation between $\Delta R$ and $s$. This is valid also for the correlation between $\Delta R$ and $p_{0}$, where more distinguishable results for both types of deformation are present. The neutron skin thickness $\Delta R$ for $\mathrm{Kr}$ isotopes correlates with $p_{0}$ almost linearly, as in the symmetry-energy case, with an inflectionpoint transition at the semimagic ${ }^{86} \mathrm{Kr}$ nucleus. In addition, one can see also from Fig. 2 that the calculated values for $p_{0}$ are smaller in the case of LNS and SLy4 forces than for the other two Skyrme parameter sets. In general, we would like to note that the behavior of deformed $\mathrm{Kr}$ isotopes shown in Fig. 2 is comparable with the one found for the spherical $\mathrm{Ni}$ and $\mathrm{Sn}$ isotopes having a magic proton number that we discussed in Ref. [24]. The small differences just indicate that stability patterns are less regular within isotopic chains with a nonmagic proton number.

For more complete study, we also consider in our work the extremely neutron-rich $\mathrm{Kr}$ isotopes $(A=96-120)$. The results for the symmetry energy $s$ as a function of the mass number $A$ for the whole $\mathrm{Kr}$ isotopic chain $(A=82-120)$ are presented in Fig. 3. We observe peaks of the symmetry energy at specific $\mathrm{Kr}$ isotopes, namely at semimagic ${ }^{86} \mathrm{Kr}(N=50)$ and ${ }^{118} \mathrm{Kr}$ $(N=82)$ nuclei. In addition, a flat area is found surrounded by transitional regions $A=88-96$ and $A=110-116$. Also, the SGII and Sk3 forces yield values of $s$ comparable with each other that lie between the corresponding symmetry energy values when using SLy4 and LNS sets. The specific nature

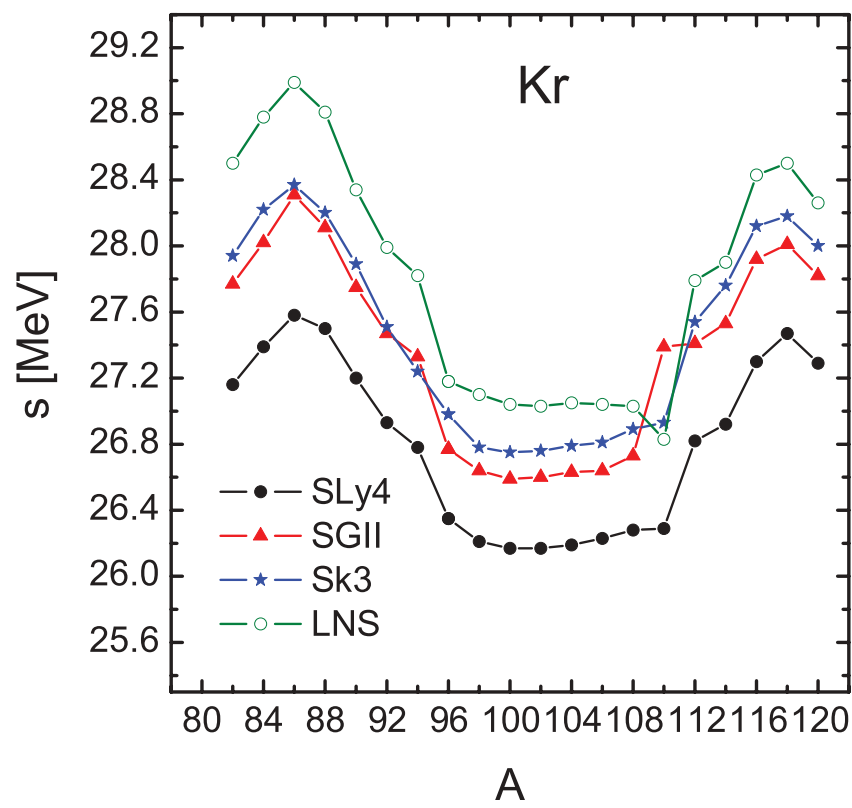

FIG. 3. (Color online) The symmetry energies $s$ for $\mathrm{Kr}$ isotopes ( $A=82-120)$ calculated with SLy4, SGII, Sk3, and LNS forces.

of the LNS force [48] (not being fitted to finite nuclei) leads to larger values of $s$ (and to a larger size of the neutron skin thickness, as is seen from Fig. 2) with respect to the results with other three forces. Although the values of $s$ slightly vary within the $\mathrm{Kr}$ isotopic chain when using different Skyrme forces, the curves presented in Fig. 3 exhibit the same trend.

The results shown in Fig. 3 are closely related to the evolution of the quadrupole parameter $\beta=\sqrt{\pi / 5} Q /\left(A\left\langle r^{2}\right\rangle^{1 / 2}\right)(Q$ being the mass quadrupole moment and $\left\langle r^{2}\right\rangle^{1 / 2}$ the nucleus rms radius) as a function of the mass number $A$ that is presented in Fig. 4. First, one can see from Fig. 4 that the semimagic $A=86$ and $A=118 \mathrm{Kr}$ isotopes are spherical, while the open-shell $\mathrm{Kr}$ isotopes within this chain possess two equilibrium shapes, oblate and prolate. In the case of open-shell isotopes, the oblate and prolate minima are very close in energy and the energy difference is always less than

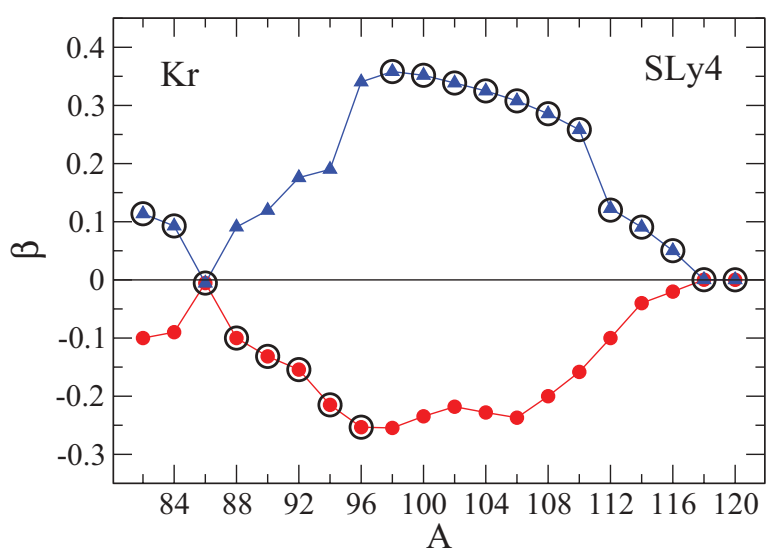

FIG. 4. (Color online) The quadrupole parameter $\beta$ as a function of the mass number $A$ for the even-even $\mathrm{Kr}$ isotopes $(A=82-120)$ in the case of the SLy4 force. 


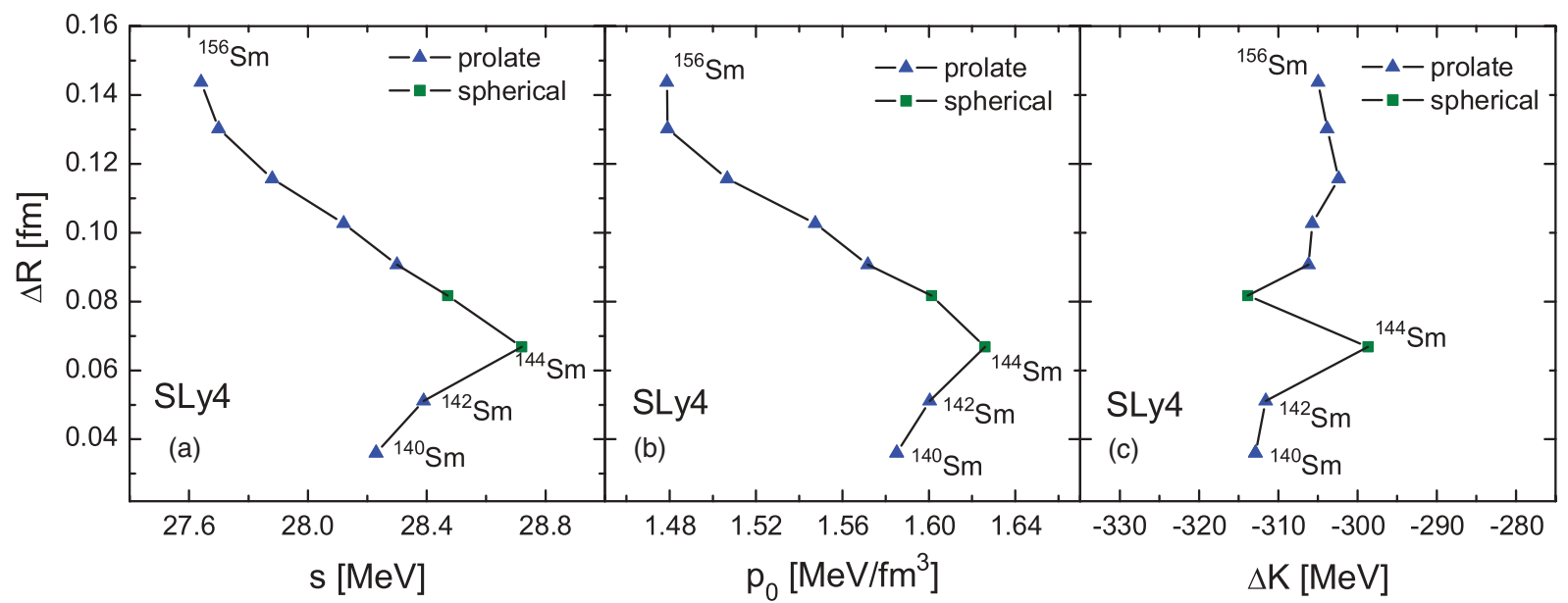

FIG. 5. (Color online) HF + BCS neutron skin thicknesses $\Delta R$ for Sm isotopes as a function of the symmetry energy $s$ (a), pressure $p_{0}$ (b), and asymmetric compressibility $\Delta K$ (c) calculated with the SLy4 force.

$1 \mathrm{MeV}$. In this region of even-even $\mathrm{Kr}$ isotopes with very large $N / Z$ ratio $(\geqslant 1.7)$ the competition between the prolate and oblate shapes has also been studied with HFB calculations and the Gogny force in Ref. [51]. Shape coexistence in lighter $\mathrm{Kr}$ isotopes has also been examined [52,53]. Nevertheless, we specify in Fig. 4 which shape corresponds to the ground state of each isotope by encircling them. Thus, the trend that the evolution of the symmetry energy shown in Fig. 3 follows can be clearly understood. The peaks of the symmetry energy correspond to the closed-shell nuclei that are spherical. Mid-shell nuclei $(A=96-110)$ are well deformed and exhibit a stabilized behavior with small values of $s$. The transitional regions from spherical to well deformed shapes correspond to transitions from the peaks to the valley in the symmetry energy.

In Figs. 5 and 6 we give results for Sm isotopes $(A=140$ 156) as a well established example of deformed nuclei. In the calculations, all $\mathrm{Sm}$ isotopes are found to have a prolate shape, except for the even-even ${ }^{144} \mathrm{Sm}$ and ${ }^{146} \mathrm{Sm}$ nuclei that are spherical. Such an evolution of shape from the spherical to the axially deformed shapes in the same $\mathrm{Sm}$ isotopic region is in accordance with the results obtained from microscopic calculations in the RMF theory [54]. In Ref. [54] the ground state of the semimagic ${ }^{144} \mathrm{Sm}(N=82)$ is found to be spherical (having about a $12 \mathrm{MeV}$ stiff barrier against deformation) and the deformation in ${ }^{146} \mathrm{Sm}$ to be still small. With the increase of the neutron number, the ground state gradually moves toward the deformed one until the well deformed ${ }^{154-158} \mathrm{Sm}$ [54]. Also, the analysis of the potential energy curves [15] within the same microscopic approach that we use in the present work, as well as within the HFB method with Gogny interaction [19], confirmed the transitional behavior between the spherical ${ }^{144} \mathrm{Sm}$ and the well prolate-deformed ${ }^{154-158} \mathrm{Sm}$ isotopes.

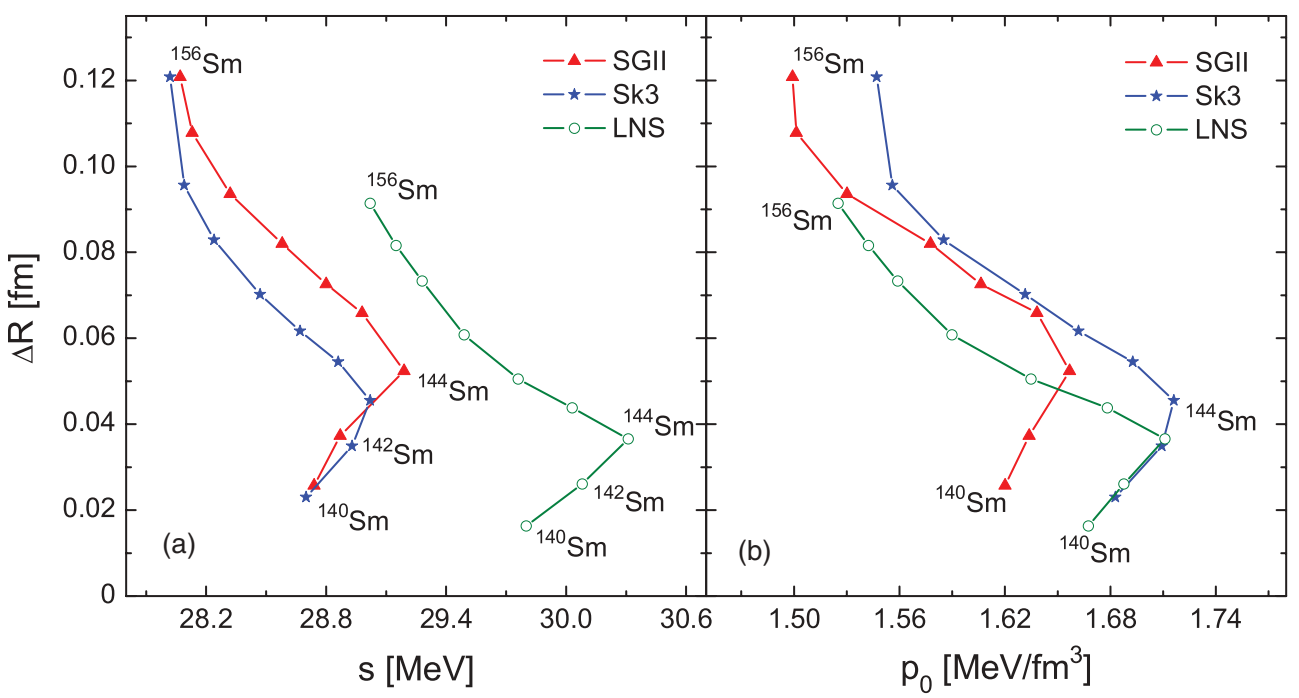

FIG. 6. (Color online) HF + BCS neutron skin thicknesses $\Delta R$ for Sm isotopes as a function of the symmetry energy $s$ (a) and the pressure $p_{0}$ (b) calculated with SGII, Sk3, and LNS forces. 


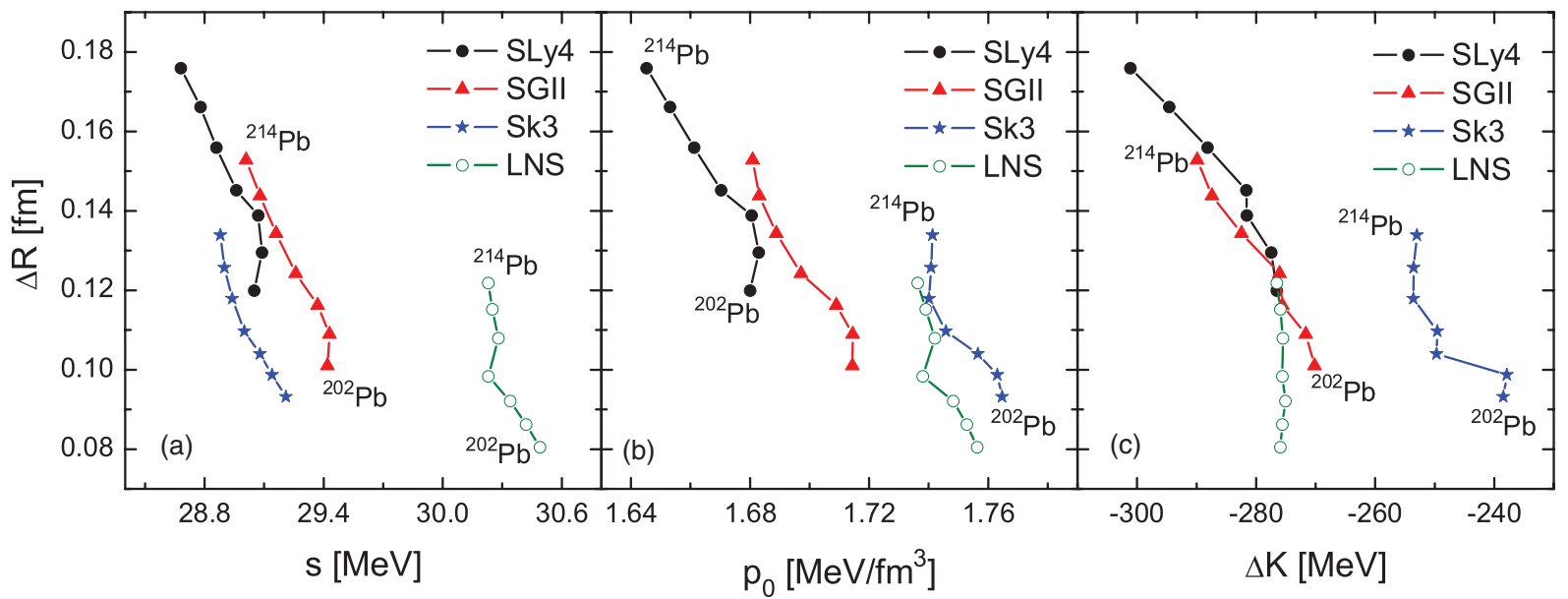

FIG. 7. (Color online) HF + BCS neutron skin thicknesses $\Delta R$ for Pb isotopes as a function of the symmetry energy $s$ (a), pressure $p_{0}$ (b), and asymmetric compressibility $\Delta K$ (c) calculated with SLy4, SGII, Sk3, and LNS forces.

The results for the correlation between the neutron skin thickness and the nuclear matter properties in finite nuclei with the SLy4 Skyrme force for a chain of Sm isotopes are shown in Fig. 5, while those with SGII, Sk3, and LNS forces are presented in Fig. 6. Similar to the case of $\mathrm{Kr}$ isotopes with transition at specific shell closure, we observe a smooth growth of the symmetry energy until the semimagic nucleus ${ }^{144} \mathrm{Sm}$ $(N=82)$ and then an almost linear decrease of $s$ while the neutron skin thickness of the isotopes increases. An approximate linear correlation between $\Delta R$ and $p_{0}$ is also shown in Figs. 5(b) and 6(b), while Fig. 5(c) exhibits a very irregular behavior of $\Delta R$ as a function of the asymmetric compressibility $\Delta K$. Nevertheless, the values of $\Delta K$ deduced from our calculations are in the interval between -295 and $-315 \mathrm{MeV}$ that compares fairly well with the neutron-asymmetry compressibility $\left(K_{\Sigma}^{\prime}=\right.$ $-320 \pm 180 \mathrm{MeV}$ ) deduced from the data [55] on the breathing mode giant monopole resonances in the isotopic chains of $\mathrm{Sm}$ and $\mathrm{Sn}$ nuclei.

The theoretical neutron skin thickness $\Delta R$ of $\mathrm{Pb}$ nuclei ( $A=202-214)$ against the parameters of interest, $s, p_{0}$, and $\Delta K$, is illustrated in Fig. 7. In this work we consider an extended chain of $\mathrm{Pb}$ isotopes in comparison to the one analyzed in Ref. [24] by adding two nuclei lighter than ${ }^{206} \mathrm{~Pb}$. Therefore, a more precise study of the corresponding correlations, especially in the transition region at the doublemagic ${ }^{208} \mathrm{~Pb}$ nucleus, could be made. Here, we test three more parametrizatios (SGII, Sk3, and LNS) in addition to the SLy4 force applied in Ref. [24]. All predicted correlations manifest an almost linear dependence and no pronounced kink at ${ }^{208} \mathrm{~Pb}$ is observed. Similarly to $\mathrm{Kr}$ and $\mathrm{Sm}$ isotopes presented in this study (and isotopes from $\mathrm{Ni}$ and $\mathrm{Sn}$ chains described in Ref. [24]), the LNS force produces larger symmetry energies $s$ than the other three forces also for $\mathrm{Pb}$ nuclei with values exceeding $30 \mathrm{MeV}$. Another peculiarity of the results obtained with LNS is the almost constant $\Delta K$ observed in Fig. 7(a).

Further attention is deserved for the value of the neutron skin thickness in ${ }^{208} \mathrm{~Pb}$, whose determination has motivated recent experiments. The model-independent measurement of parity-violating asymmetry (which is sensitive to the neutron distribution) in the elastic scattering of polarized electrons from ${ }^{208} \mathrm{~Pb}$ at JLAB by the PREX Collaboration $[27,28]$ has provided the first electroweak observation of $\Delta R=$ $0.33_{-0.18}^{+0.16} \mathrm{fm}$ in ${ }^{208} \mathrm{~Pb}$. Obviously, future precise measurements are needed to reduce the quoted uncertainties of $\Delta R$. The distorted-wave electron scattering calculations for ${ }^{208} \mathrm{~Pb}$ [31] extracted a result for the neutron skin thickness which agrees with that reported in the experimental paper [28]. As can be seen from Fig. 7(a), the value of $\Delta R$ for ${ }^{208} \mathrm{~Pb}(0.1452 \mathrm{fm})$ deduced from the present HF + BCS calculations with the SLy4 force agrees with the recent experimentally extracted skin thickness $\left(0.156_{-0.021}^{+0.025} \mathrm{fm}\right)$ using its correlation with the dipole polarizability [29]. However, this experimental value was derived by means of covariance analysis based on one Skyrme functional (SV-min). In this respect, a systematic study with a variety of EDFs as well as experimental tests in other nuclei would be important because the correlation between polarizability, neutron skin thickness, and symmetry energy is model dependent (see, for example, Fig. 1 of Ref. [33]). In addition, our theoretically obtained value of $\Delta R$ for ${ }^{208} \mathrm{~Pb}$ agrees well with the value $0.18 \pm 0.027 \mathrm{fm}$ from Ref. [40]. It is lower than the one obtained in Refs. [56,57] with the same Skyrme force, but is in agreement with the values calculated with self-consistent densities of several nuclear mean-field models (see Table I in Ref. [58]). The $p_{0}$ and $\Delta K$ values for ${ }^{208} \mathrm{~Pb}$ are in a good agreement with those from Ref. [56].

We would like to note that linear correlations $\Delta R$ vs $s$ and $\Delta R$ vs $p_{0}$ are found to exist in the $\mathrm{Kr}(A=82-96), \mathrm{Sm}$ ( $A=140-156)$, and $\mathrm{Pb}(A=202-214)$ isotopic chains. The correlation $\Delta R$ vs $\Delta K$ presented for the examples of $\mathrm{Sm}$ and $\mathrm{Pb}$ isotopes (Figs. 5 and 7, respectively) is less strong than the ones mentioned before and shows a slight irregular behavior. This concerns also the calculated results for $\Delta K$ in the case of $\mathrm{Kr}$ isotopes that are not shown in Fig. 2. Such observations are confirmed by the results for these correlations obtained in Refs. [59,60]. In addition, it was demonstrated in Ref. [61] that, among various observables that correlate with the neutron form factor (related to the neutron density and, thus, to the neutron 


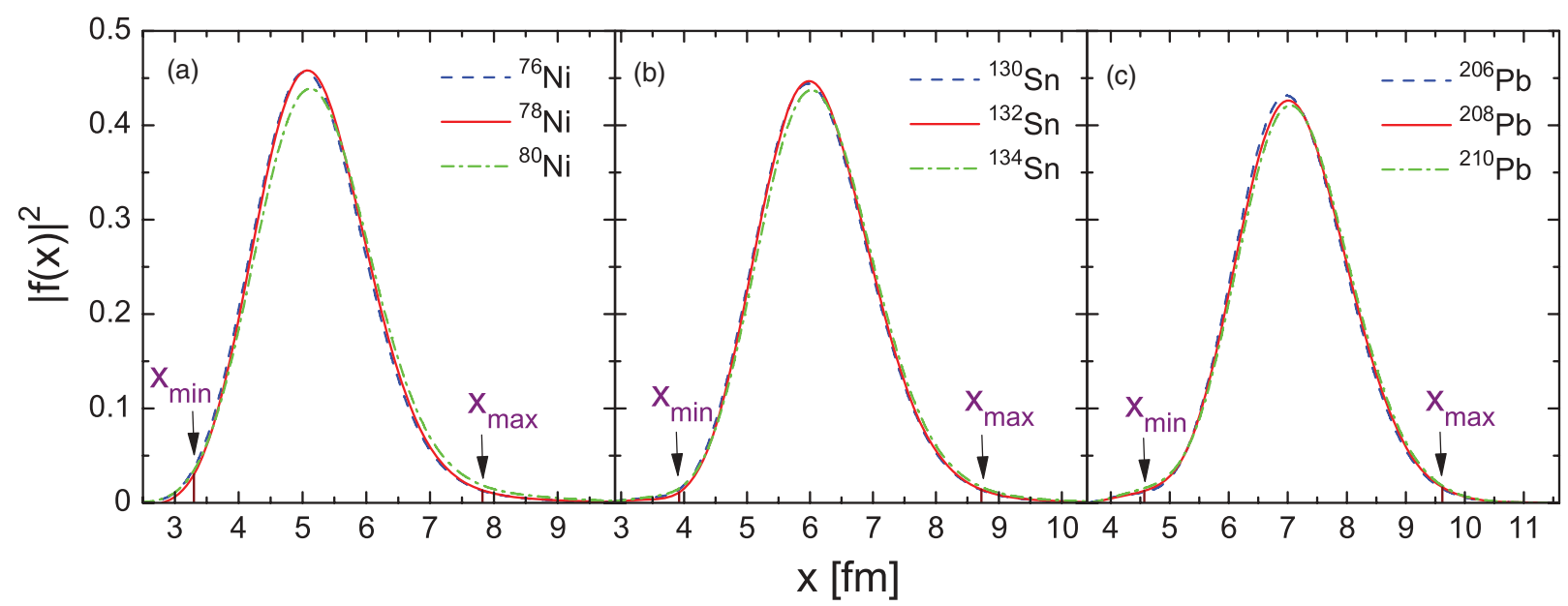

FIG. 8. (Color online) The weight function $|f(x)|^{2}$ calculated for three Ni $(A=76,78,80)$ isotopes (a), three $\mathrm{Sn}(A=130,132,134)$ isotopes (b), and three $\mathrm{Pb}(A=206,208,210)$ isotopes (c) by using the HF + BCS total densities for these nuclei and with the SLy4 force.

skin thickness), the incompressibility is a poor indicator of isovector properties.

Following our previous analysis within the CDFM approach [24], here we would like to give more detailed study of the weight function $|f(x)|^{2}$ (that is related to the density and thus, to the structural peculiarities) to understand the kinks observed in the relationships between $\Delta R$ and $s$, as well as $\Delta R$ and $p_{0}$. The latter were shown to exist [24] in double-magic nuclei in the cases of $\mathrm{Ni}\left(\right.$ at $\left.{ }^{78} \mathrm{Ni}\right)$ and $\mathrm{Sn}\left(\right.$ at $\left.{ }^{132} \mathrm{Sn}\right)$ isotopic chains. As one can see in Figs. 2, 5, and 6 of the present work, they exist also in the considered cases of $\mathrm{Kr}\left(\right.$ at $\left.{ }^{86} \mathrm{Kr}\right)$ and $\mathrm{Sm}\left(\right.$ at $\left.{ }^{144} \mathrm{Sm}\right)$ isotopes. In contrast, such a kink does not exist in the case of the $\mathrm{Pb}$ isotopic chain (at ${ }^{208} \mathrm{~Pb}$, particularly).

Here we analyze, as an example, the cases of $\mathrm{Ni}, \mathrm{Sn}$, and $\mathrm{Pb}$ isotopic chains, trying to understand the origin of the kinks without additional complexities coming from deformation. For this purpose, let us introduce the quantity

$$
\Delta s_{ \pm}=\frac{s_{A \pm 2}-s_{A}}{s_{A}}
$$

which gives information on the relative deviation of the symmetry energy $s$ of even-even isotopes with respect to the double-magic ones, namely with $A=78$ for Ni, $A=132$ for $\mathrm{Sn}$, and $A=208$ for $\mathrm{Pb}$. Here we will consider the range of integration on $x$ in Eq. (10) in each of the cases of $\mathrm{Ni}, \mathrm{Sn}$, and $\mathrm{Pb}$ isotopes. First, we will introduce the value of $x_{\min }$ at which the symmetry energy for nuclear matter $s^{\mathrm{ANM}}(x)$ changes sign from negative (at $x<x_{\min }$ ) to positive (at $x>x_{\min }$ ). In our work we use the symmetry energy $s^{\mathrm{ANM}}(\rho(x))$ [Eq. (18)] from the Brueckner theory, that is defined by the second derivative of the energy per particle $E(\rho, \delta)$. Considering in principle in the CDFM the range of $x$ from zero to infinity we include in this way the region of densities $\rho_{0}(x)$ from infinity to zero, respectively. When the values of $x$ are small we consider in practice values of the density $\rho_{0}(x)$ that are much larger than the density in the equilibrium state $\rho_{0}$. In this case unphysical (negative) values of the symmetry energy appear, and thus starting the integration from $x \geqslant x_{\min }$ we exclude these values. We have to note simultaneously that at $x<x_{\min }$ the weight function $|f(x)|^{2}$ is close to zero (it is its "left wing"; see Fig. 8), so there is no contribution to $s$ from this region $\left(x<x_{\min }\right)$. Secondly, we introduce the value of $x_{\max }$ (in the "right wing" of $|f(x)|^{2}$ ) beyond which the contribution to $s$ [i.e., the result of the integration in Eq. (10) from $x_{\max }$ to infinity] is negligible. If we define by $\Delta x=x_{\max }-x_{\min }$, then we impose for $x_{\max }$ the condition $s-s_{\Delta x} \leqslant 0.1 \mathrm{MeV}$, where $s_{\Delta x}$ is obtained by Eq. (10) integrating over $x$ from $x_{\min }$ to $x_{\max }$, while $s$ is the result of integration from $x_{\min }$ to infinity. The points $x_{\min }$ and $x_{\max }$ corresponding to the double-magic nuclei of the three isotopic chains considered are indicated in Fig. 8, where the intrinsic weight functions $|f(x)|^{2}$ for ${ }_{76,78,80} \mathrm{Ni}$ [Fig. 8(a)], ${ }^{130,132,134} \mathrm{Sn}$ [Fig. 8(b)], and ${ }^{206,208,210} \mathrm{~Pb}$ [Fig. 8(c)] are separately presented. Then, if one uses Eq. (10) that determines the symmetry energy $s$ in finite nuclei within the CDFM, the contribution $s_{\Delta x}$ of $s$ in the interval $\Delta x$ is obtained. The values of $s_{\Delta x}$ are given in Table I together with

TABLE I. Integration limit values $x_{\min }$ and $x_{\max }$ (in $\mathrm{fm}$ ) and values of the contribution $s_{\Delta x}$ to the total symmetry energy $s$ (in MeV) for three $\mathrm{Ni}(A=76,78,80), \mathrm{Sn}(A=130,132,134)$, and $\mathrm{Pb}(A=206,208,210)$ isotopes.

\begin{tabular}{|c|c|c|c|c|c|c|c|c|c|}
\hline & ${ }^{76} \mathrm{Ni}$ & ${ }^{78} \mathrm{Ni}$ & ${ }^{80} \mathrm{Ni}$ & ${ }^{130} \mathrm{Sn}$ & ${ }^{132} \mathrm{Sn}$ & ${ }^{134} \mathrm{Sn}$ & ${ }^{206} \mathrm{~Pb}$ & ${ }^{208} \mathrm{~Pb}$ & ${ }^{210} \mathrm{~Pb}$ \\
\hline$x_{\min }$ & 3.26 & 3.30 & 3.32 & 3.90 & 3.92 & 3.94 & 4.56 & 4.56 & 4.58 \\
\hline$x_{\max }$ & 7.78 & 7.82 & 8.16 & 8.70 & 8.72 & 8.92 & 9.56 & 9.62 & 9.64 \\
\hline$s$ & 27.65 & 27.85 & 27.47 & 28.62 & 28.76 & 28.56 & 29.07 & 28.96 & 28.86 \\
\hline
\end{tabular}




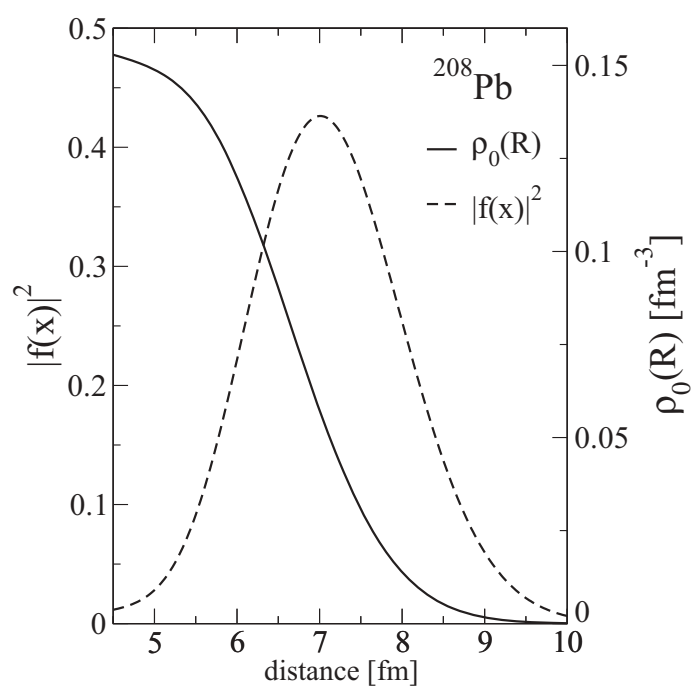

FIG. 9. The weight function $|f(x)|^{2}$ and the $\mathrm{HF}+\mathrm{BCS}$ total density $\rho_{0}(R)$ of ${ }^{208} \mathrm{~Pb}$ calculated with the SLy4 force.

the values of $x_{\min }, x_{\max }$, and $s$. We can see from Table I that, as can be expected, the total symmetry energy is almost exhausted by its contribution $s_{\Delta x}$, thus showing the important role which the nuclear surface plays for the obtained symmetry energy values. For a better illustration of this fact, we plot together in Fig. 9 the weight function $|f(x)|^{2}$ and the HF + BCS total density $\rho_{0}(R)$ of ${ }^{208} \mathrm{~Pb}$ in the case of the SLy4 force. As can be seen, the nuclear surface part of the density which determines the weight function $|f(x)|^{2}$ containing the peak around the maximum is responsible to a large extent for the main contribution to the total symmetry energy.
TABLE II. Relative deviation values of the symmetry energy $\Delta s_{+}$ and $\Delta s_{-}$[Eq. (30)] for the range of integration $\Delta x$ in Eq. (10) and for $\mathrm{Ni}, \mathrm{Sn}$, and $\mathrm{Pb}$ isotopes.

\begin{tabular}{lccr}
\hline \hline & $\mathrm{Ni}$ & $\mathrm{Sn}$ & \multicolumn{1}{c}{$\mathrm{Pb}$} \\
\hline$\Delta s_{+}$ & -0.0137 & -0.0070 & -0.0035 \\
$\Delta s_{-}$ & -0.0072 & -0.0049 & 0.0038 \\
\hline \hline
\end{tabular}

The analysis of the quantity $\Delta s_{ \pm}$[Eq. (30)] might be instructive because this quantity is a direct measure of the relative deviation of the symmetry energy with respect to the double-magic nuclei, taking them as reference nuclei in each of the chains, where the kinks are expected. The values of $\Delta s_{+}$ and $\Delta s_{-}$are listed in Table II, where the two numbers for each isotopic chain correspond to the range of integration $\Delta x$. One can see first from this table that the absolute values of $\Delta s_{+}$and $\Delta s_{-}$for $\mathrm{Pb}$ isotopes are comparable with each other, which is not the case for the two other isotopic chains. Second, and very important, is that the $\Delta s_{+}$value turns out to be negative and the $\Delta s_{-}$value to be positive for $\mathrm{Pb}$ isotopes in the range of integration $\Delta x$, and this is the main difference regarding the corresponding values (both are negative) in the $\mathrm{Ni}$ and $\mathrm{Sn}$ chains.

These differences can be attributed to the profiles of the density distributions, particularly in the surface region. They are given in Fig. 10, where curves for five $\mathrm{Ni}, \mathrm{Sn}$, and $\mathrm{Pb}$ isotopes around double-magic ${ }^{78} \mathrm{Ni},{ }^{132} \mathrm{Sn}$, and ${ }^{208} \mathrm{~Pb}$ nuclei are presented in panels (a), (b), and (c), respectively. One can see from Fig. 10 the same trend in the tails of the three isotopic chains, which are ordered according to the mass number $A$, being higher for heavier isotopes to produce larger radii. On the other hand the behavior in the top part of the surface

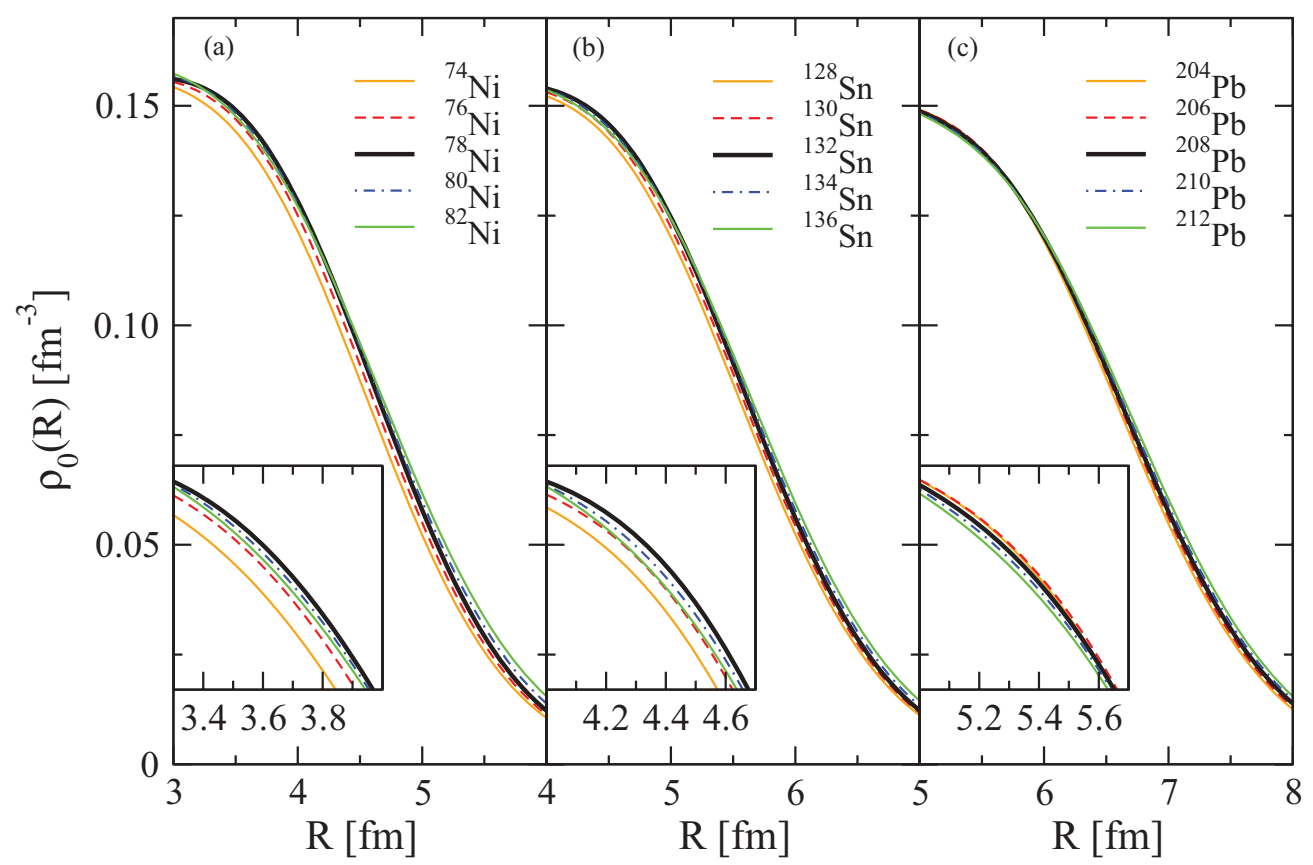

FIG. 10. (Color online) HF + BCS total densities in the surface region for five Ni $(A=74-82)$ isotopes $(a)$, five Sn $(A=128-136)$ isotopes (b), and five $\mathrm{Pb}(A=204-212)$ isotopes (c) around double-magic ${ }^{78} \mathrm{Ni},{ }^{132} \mathrm{Sn}$, and ${ }^{208} \mathrm{~Pb}$ nuclei, respectively, calculated with the SLy4 force. 
region, shown in the inset of the panels, is different. In the case of $\mathrm{Ni}$ and $\mathrm{Sn}$ isotopes in panels (a) and (b), one observes that the double-magic nuclei have the largest density with all the neighboring isotopes lying below. In the case of $\mathrm{Pb}$ isotopes in panel (c), the density increases from heavier to lighter isotopes with the double-magic nucleus in between. In $\mathrm{Pb}$ isotopes, this ordering is opposite in the tail. As a result of this, the slope of the density in $\mathrm{Pb}$ isotopes, and therefore $|f(x)|^{2}$, decreases with the number of neutrons continuously and no kink is present in the symmetry energy. On the other hand, in the case of $\mathrm{Ni}$ and $\mathrm{Sn}$ isotopes, the slope of the density is larger for the double-magic isotopes, generating a kink in the symmetry energy.

In this way, the kinks displayed in our previous study [24] by the $\mathrm{Ni}$ and $\mathrm{Sn}$ isotopes and the fact that no kinks appear in the $\mathrm{Pb}$ chain considered can be understood. It concerns not only the symmetry energy evolution, but also its relationship with the neutron skin thickness $\Delta R$ and the pressure $p_{0}$. We would like to summarize the discussion about the kinks, saying that within the CDFM we are able to use only the densities resulting from the specific shells that are occupied. The presented Figs. 9 and 10 give information about the differences between densities of $\mathrm{Ni}, \mathrm{Sn}$, and $\mathrm{Pb}$ isotopes that are reflected in the corresponding weight functions $|f(x)|^{2}$ for these isotopes (see Fig. 8). Of course, these differences are due to the different occupancies of the shells in these nuclei. Thus, we see that in general kinks are produced at shell closures, but the analyses of the precise dependence of various kinks on the amount of occupation of specific shells will require further work.

\section{CONCLUSIONS}

In this paper, we have investigated possible relationships between the neutron skin thickness of deformed neutron-rich nuclei and the symmetry energy characteristics of nuclear matter for these nuclei. A microscopic approach based on deformed $\mathrm{HF}+$ BCS calculations with Skyrme forces has been used. Four Skyrme parametrizations were involved in the calculations: SGII, Sk3, SLy4, and LNS. Nuclear matter properties of nuclei from $\mathrm{Kr}$ and $\mathrm{Sm}$ isotopic chains have been studied by applying the CDFM that provides a transparent and analytic way to calculate the intrinsic EOS quantities by means of a convenient approach to the weight function. As a first step we study the variation with neutron number of proton and neutron radii predicted by the self-consistent microscopic $(\mathrm{HF}+\mathrm{BCS})$ calculations. We find that charge and proton radii increase similarly with neutron number following the increase of neutron radii, as it should be expected from the general properties of the nuclear force. The same microscopic method is then applied to calculate the skin thickness and the weight function for each isotope. The analysis of the nuclear symmetry energy $s$, the neutron pressure $p_{0}$, and the asymmetric compressibility $\Delta K$ has been carried out on the basis of the Brueckner EDF for infinite nuclear matter.

For both $\operatorname{Kr}(A=82-96)$ and $\operatorname{Sm}(A=140-156)$ isotopic chains we have found that there exists an approximate linear correlation between the neutron skin thickness of these nuclei and their nuclear symmetry energies. Comparing with the spherical case of $\mathrm{Ni}, \mathrm{Sn}$, and $\mathrm{Pb}$ nuclei described in our previous study [24], we note that the linear correlation observed in the $\mathrm{Kr}$ and $\mathrm{Sm}$ isotopes is not smooth enough due to their different equilibrium shapes, as well as to the transition regions between them. As known, the latter are difficult interpret as they exhibit a complicated interplay of competing degrees of freedom. Nevertheless, a smoother behavior is observed in $\mathrm{Kr}$ isotopes that is a consequence of the stabilization of the oblate shapes along the isotopic chain. As far as $\mathrm{Sm}$ isotopes are concerned, the shape evolution from the spherical to the axially deformed configurations in the Sm isotopes causes a less pronounced linearity of the observed correlation between $\Delta R$ and $s$. A similar correlation between $\Delta R$ and $p_{0}$ is also found to exist, while the relation between $\Delta R$ and $\Delta K$ exhibits an irregular behavior. However, for both classes of deformed nuclei an inflection-point transition at specific shell closure, in particular at semimagic ${ }^{86} \mathrm{Kr}$ and ${ }^{144} \mathrm{Sm}$ nuclei, appears for these correlations of the neutron skins with $s$ and $p_{0}$. In addition, the role of the relative neutron-proton asymmetry on the evolution of the symmetry energy has been pointed out on the example of $\mathrm{Kr}$ isotopes with $N>60$.

We have analyzed in detail the existence of kinks on the example of the $\mathrm{Ni}$ and $\mathrm{Sn}$ isotopic chains and the lack of such a kink for the $\mathrm{Pb}$ isotopic chain. For this purpose, we have estimated the relative deviation of the symmetry energy of even-even isotopes with respect to the double-magic ${ }^{78} \mathrm{Ni}$, ${ }^{132} \mathrm{Sn}$, and ${ }^{208} \mathrm{~Pb}$ nuclei. An analysis of the weight function $|f(x)|^{2}$ (determined by the HF + BCS density distributions) as the key ingredient of the CDFM is performed. It is shown that for the $\mathrm{Pb}$ isotopes the different signs of the relative deviations, corresponding to the range of integration on $x$ in Eq. (10) that contains the peak of $|f(x)|^{2}$, is in favor of the absence of a kink in the $\mathrm{Pb}$ chain. Thus, from the previous study in Ref. [24] and the present analysis, the kinks displayed by $\mathrm{Ni}$ and $\mathrm{Sn}$ can be understood as consequences of particular differences in the structure of these nuclei and the resulting densities and weight functions.

It has to be mentioned that the used microscopic theoretical approach is capable also of predicting important nuclear matter quantities in deformed neutron-rich exotic nuclei and their relation to surface properties of these nuclei. This is confirmed by the good agreement achieved with other theoretical predictions and some experimentally extracted ground-state properties. New experimental results on giant resonances, neutron skin in heavy nuclei, and heavy-ion collisions could further lead to new constraints, e.g., on the nuclear symmetry energy, allowing successful interpretation of data and observations on physical quantities of nuclear systems.

\section{ACKNOWLEDGMENTS}

Two of the authors (M.K.G. and A.N.A.) are grateful for the support of the Bulgarian Science Fund under Contract No. 02-285. E.M.G. and P.S. acknowledge support from MINECO (Spain) under Contracts No. FIS2011-23565 and No. FPA2010-17142. 
[1] P. Danielewicz, R. Lacey, and W. G. Lynch, Science 298, 1592 (2002).

[2] M. A. Famiano et al., Phys. Rev. Lett. 97, 052701 (2006).

[3] D. V. Shetty, S. J. Yennello, and G. A. Souliotis, Phys. Rev. C 76, 024606 (2007).

[4] M. Centelles, X. Roca-Maza, X. Viñas, and M. Warda, Phys. Rev. Lett. 102, 122502 (2009).

[5] B. A. Brown, Phys. Rev. Lett. 85, 5296 (2000).

[6] S. Typel and B. A. Brown, Phys. Rev. C 64, 027302 (2001).

[7] C. J. Horowitz and J. Piekarewicz, Phys. Rev. Lett. 86, 5647 (2001).

[8] J. M. Lattimer and M. Prakash, Phys. Rep. 442, 109 (2007).

[9] V. Baran, M. Colonna, V. Greco, and M. Di Toro, Phys. Rep. 410, 335 (2005).

[10] J. L. Wood, K. Heyde, W. Nazarewicz, M. Huyse, and P. Van Duppen, Phys. Rep. 215, 101 (1992); K. Heyde and J. L. Wood, Rev. Mod. Phys. 83, 1467 (2011).

[11] M. V. Stoitsov, J. Dobaczewski, W. Nazarewicz, S. Pittel, and D. J. Dean, Phys. Rev. C 68, 054312 (2003).

[12] S. Goriely, N. Chamel, and J. M. Pearson, Phys. Rev. Lett. 102, 152503 (2009).

[13] D. Vautherin, Phys. Rev. C 7, 296 (1973).

[14] M. Bender, P.-H. Heenen, and P.-G. Reinhard, Rev. Mod. Phys. 75, 121 (2003).

[15] R. Rodríguez-Guzmán and P. Sarriguren, Phys. Rev. C 76, 064303 (2007); P. Sarriguren, R. Rodríguez-Guzmán, and L. M. Robledo, ibid. 77, 064322 (2008).

[16] P. Sarriguren, M. K. Gaidarov, E. M. de Guerra, and A. N. Antonov, Phys. Rev. C 76, 044322 (2007).

[17] M. K. Gaidarov, G. Z. Krumova, P. Sarriguren, A. N. Antonov, M. V. Ivanov, and E. Moya de Guerra, Phys. Rev. C 80, 054305 (2009).

[18] J. Dechargé and D. Gogny, Phys. Rev. C 21, 1568 (1980).

[19] L. M. Robledo, R. R. Rodríguez-Guzmán, and P. Sarriguren, Phys. Rev. C 78, 034314 (2008).

[20] Z. Sheng, Z. Ren, and W. Jiang, Nucl. Phys. A 832, 49 (2010).

[21] Z. Sheng and Z. Ren, Eur. Phys. J. A 46, 241 (2010).

[22] N. Nikolov, N. Schunck, W. Nazarewicz, M. Bender, and J. Pei, Phys. Rev. C 83, 034305 (2011).

[23] T. Nikšić, D. Vretenar, and P. Ring, Phys. Rev. C 78, 034318 (2008).

[24] M. K. Gaidarov, A. N. Antonov, P. Sarriguren, and E. Moya de Guerra, Phys. Rev. C 84, 034316 (2011).

[25] A. N. Antonov, V. A. Nikolaev, and I. Zh. Petkov, Bulg. J. Phys. 6, 151 (1979); Z. Phys. A 297, 257 (1980); 304, 239 (1982); Nuovo Cimento A 86, 23 (1985); A. N. Antonov et al., ibid. 102, 1701 (1989); A. N. Antonov, D. N. Kadrev, and P. E. Hodgson, Phys. Rev. C 50, 164 (1994).

[26] A. N. Antonov, P. E. Hodgson, and I. Zh. Petkov, Nucleon Momentum and Density Distributions in Nuclei (Clarendon, Oxford, 1988); Nucleon Correlations in Nuclei (Springer-Verlag, Berlin, 1993).

[27] [http://hallaweb.jlab.org/parity/prex].

[28] S. Abrahamyan et al., Phys. Rev. Lett. 108, 112502 (2012).

[29] A. Tamii et al., Phys. Rev. Lett. 107, 062502 (2011).

[30] O. Moreno, E. Moya de Guerra, P. Sarriguren, and J. M. Udias, J. Phys. G 37, 064019 (2010).
[31] C. J. Horowitz et al., Phys. Rev. C 85, 032501(R) (2012).

[32] X. Roca-Maza, M. Centelles, X. Viñas, and M. Warda, Phys. Rev. Lett. 106, 252501 (2011).

[33] J. Piekarewicz, B. K. Agrawal, G. Colò, W. Nazarewicz, N. Paar, P.-G. Reinhard, X. Roca-Maza, and D. Vretenar, Phys. Rev. C 85, 041302(R) (2012).

[34] M. Warda, X. Viñas, X. Roca-Maza, and M. Centelles, Phys. Rev. C 81, 054309 (2010).

[35] A. Z. Mekjian and L. Zamick, Phys. Rev. C 85, 057303 (2012).

[36] A. E. L. Dieperink, Y. Dewulf, D. Van Neck, M. Waroquier, and V. Rodin, Phys. Rev. C 68, 064307 (2003).

[37] L.-W. Chen, Phys. Rev. C 83, 044308 (2011).

[38] P. Danielewicz and J. Lee, in VII Latin American Symposium on Nuclear Physics and Applications, AIP Conf. Proc. No. 947 (AIP, New York, 2007), p. 301; Nucl. Phys. A 818, 36 (2009).

[39] A. Klimkiewicz et al., Phys. Rev. C 76, 051603(R) (2007).

[40] M. B. Tsang et al., e-print arXiv:1204.0466 [nucl-ex].

[41] K. A. Brueckner, J. R. Buchler, S. Jorna, and R. J. Lombard, Phys. Rev. 171, 1188 (1968).

[42] K. A. Brueckner, J. R. Buchler, R. C. Clark, and R. J. Lombard, Phys. Rev. 181, 1543 (1969).

[43] G. Audi, A. H. Wapstra, and C. Thibault, Nucl. Phys. A 729, 337 (2003).

[44] E. Moya de Guerra, P. Sarriguren, J. A. Caballero, M. Casas, and D. W. L. Sprung, Nucl. Phys. A 529, 68 (1991).

[45] E. Chabanat, P. Bonche, P. Haensel, J. Meyer, and R. Schaeffer, Nucl. Phys. A 635, 231 (1998).

[46] M. Beiner, H. Flocard, N. Van Giai, and P. Quentin, Nucl. Phys. A 238, 29 (1975).

[47] N. Van Giai and H. Sagawa, Phys. Lett. B 106, 379 (1981).

[48] L. G. Cao, U. Lombardo, C. W. Shen, and N. V. Giai, Phys. Rev. C 73, 014313 (2006).

[49] M. Keim, E. Arnold, W. Borchers, U. Georg, A. Klein, R. Neugart, L. Vermeeren, R. E. Silverans, and P. Lievens, Nucl. Phys. A 586, 219 (1995).

[50] R. Rodríguez-Guzmán, P. Sarriguren, and L. M. Robledo, Phys. Rev. C 83, 044307 (2011).

[51] S. Hilaire and M. Girod, Eur. Phys. J. A 33, 237 (2007).

[52] P. Sarriguren, E. Moya de Guerra, and A. Escuderos, Nucl. Phys. A 658, 13 (1999); Phys. Rev. C 64, 064306 (2001).

[53] H. Flocard, P. Quentin, A. K. Kerman, and D. Vautherin, Nucl. Phys. A 203, 433 (1973).

[54] J. Meng, W. Zhang, S.-G. Zhou, H. Toki, and L. S. Geng, Eur. Phys. J. A 25, 23 (2005).

[55] M. M. Sharma, W. T. A. Borghols, S. Brandenburg, S. Crona, A. van der Woude, and M. N. Harakeh, Phys. Rev. C 38, 2562 (1988).

[56] L.-W. Chen, C. M. Ko, and B.-A. Li, Phys. Rev. C 72, 064309 (2005).

[57] B. K. Agrawal, Phys. Rev. C 81, 034323 (2010).

[58] M. Centelles, X. Roca-Maza, X. Viñas, and M. Warda, Phys. Rev. C 82, 054314 (2010).

[59] I. Vidaña, C. Providencia, A. Polls, and A. Rios, Phys. Rev. C 80, 045806 (2009).

[60] S. Yoshida and H. Sagawa, Phys. Rev. C 69, 024318 (2004).

[61] P.-G. Reinhard and W. Nazarewicz, Phys. Rev. C 81, 051303(R) (2010). 